\title{
Top-Down Influences of Spatial Attention in Visual Cortex
}

\author{
Seth E. Bouvier \\ Princeton Neuroscience Institute, Princeton University, Princeton, New Jersey 08540 \\ Review of Bressler et al. (http://www.jneurosci.org/cgi/content/full/28/40/10056)
}

It has been known for decades that neurons in visual cortex respond more vigorously when attention is directed to their receptive field. In recent years, substantial progress has been made toward understanding the neural mechanisms involved in controlling spatial attention. Many studies have shown that activity increases in several regions outside visual cortex when subjects select a spatial location for further processing. Two regions in particular, the frontal eye fields (FEFs) and the intraparietal sulcus (IPS), are consistently activated during top-down control of attention.

Considerable evidence suggests that the FEFs and IPS are responsible for the changes in visual cortical activity observed during spatial attention. The most compelling evidence comes from studies recording from neurons in monkey cortex. However, demonstrating these influences in humans under physiological conditions is difficult, in part due to inherent technical limitations of the primary neuroimaging methodology, functional magnetic resonance imaging (fMRI). Because of its poor temporal resolution, fMRI analyses typically show areas that are coactivated during a task, but not how they influence each other.

Recently, several methods of inferring functional connections between brain regions have been advanced for use with

Received Nov. 21, 2008; revised Jan. 2, 2009; accepted Jan. 6, 2009.

Correspondence should be addressed to Seth E. Bouvier, Princeton Neuroscience Institute, Princeton University, Princeton, NJ 08540. E-mail: sbouvier@princeton.edu.

DOI:10.1523/JNEUROSCI.5611-08.2009

Copyright $\odot$ 2009 Society for Neuroscience $\quad$ 0270-6474/09/291597-02\$15.00/0
fMRI data, including Granger causality (Roebroeck et al., 2005). Granger causality is a concept that was developed by Clive Granger in the 1960s for use in economic forecasting. The premise is that if prior states of process $A$ can improve predictions of the current state of process $B$, then $A$ may cause $B$. This definition of causality, based on temporal precedence and predictability, is called Granger causality to distinguish it from other definitions of causality.

Applied to fMRI data, Granger causality can be used to test the influence of one brain area (source) on another (target). Because the signal measured by fMRI is blurred in time, prior states of the target area are likely to have predictive value for its current state. The critical test of Granger causality is to what extent does adding the prior state of the source brain region improve the ability to predict the response of the target area from its own prior states. In other words, if the activity in brain area $S$ at time $t-1$ improves the ability to predict the activity in area $T$ at time $t$ compared with predictions based solely on the activity in area $T$ at time $t-$ 1 , then $S$ Granger causes $T$.

In a recent article in this journal, Bressler et al. (2008) used Granger causality measures between fMRI time series in FEFs, IPS, and visual cortex as subjects directed spatial attention. Subjects heard an auditory cue that indicated (with $75 \%$ validity) on which side of the screen they would perform an orientation discrimination task. After a variable delay, two simple stimuli appeared briefly in the upper visual field, one on either side of fixa- tion. Subjects heard a second auditory cue simultaneously with the appearance of the stimuli. Subjects reported which of three possible orientations was present in the location indicated by the second auditory cue. This task was previously shown to increase the activity of neurons in visual cortex with receptive fields at the attended location (Sylvester et al., 2007).

In a heroic effort, six subjects were scanned 16-24 times each. Granger causality was assessed at the time point just before the stimulus appeared, between regions of interest (ROIs) in frontal, parietal, and visual cortex. The critical measurement was the fraction of all voxel pairs between two ROIs that had statistically significant Granger causality. In nearly every ROI pair, the authors found a larger fraction of voxel pairs with significant top-down rather than bottom-up Granger causality. That is, for an ROI pair such as right V1 and right FEFs, Granger causality from the higher-order region (rFEFs) to the lower-order region ( $\mathrm{rV} 1$ ) was statistically significant in more voxels than in the other direction. Overall, $\sim 20 \%$ of voxel pairs showed significant Granger causality in the top-down direction compared with $10 \%$ in the bottom-up direction. Comparing the responses between the frontal and parietal regions, more voxels showed significant Granger causality from the FEFs to the IPS ROIs than in the reverse direction. While the effect was smaller than the top-down effect in visual cortex, it was present in five of six subjects. The results of this study provide good evidence that the activity of neurons in the FEFs and IPS regions pre- 
dict attention-related changes in visual cortex activity.

The results also address an ongoing debate about the nature of the circuitry between the attentional control network and visual areas. Specifically, do the frontal areas or the parietal areas control the attention-related changes in visual cortex activity? In one view, top-down attentional control begins in frontal cortex, and in the alternate view, top-down attentional control begins in parietal cortex. Two recent papers used techniques with better temporal resolution than fMRI to examine the order of frontal and parietal activity during attention, and reached opposite conclusions. In one of the two studies, the source of top-down attentional signals was localized in frontal cortex (Grent-'t-Jong and Woldorff, 2007). Yet in the other study, the responses in parietal areas preceded those in frontal areas (Green and McDonald, 2008).

Unfortunately, understanding the neural mechanisms of attentional control may be more complicated than determining whether the frontal regions precede the parietal regions or vice versa. For example, Green and McDonald (2008) conclude that their data support a model in which two parietal regions become active first and influence the activity in frontal regions. The frontal regions project back to one of the parietal regions, which projects to visual cortex. In their account, parietal regions are active twice: peaking 150-200 ms after a cue and again, beginning 600-700 ms after the cue and persisting through the anticipatory period. In contrast, the frontal regions were active once, 300-600 ms after the cue.

Because the Granger causality measured by Bressler et al. (2008) is greater from the FEFs to the IPS than from the IPS to the FEFs, the results appear to support a model in which attention is first controlled by frontal cortical regions. However, because the effect is small, interpretation is difficult. Factors beside strength of influence affect the ability to detect Granger causality, such as latency (Roebroeck et al., 2005). It is therefore possible that the IPS influences the FEFs equally, but the influence is harder to detect due to a shorter latency. Furthermore, it is unclear to what extent Granger causality analyses of time series sampled at $0.5 \mathrm{~Hz}$ are sensitive to connections like those reported by Green and McDonald (2008).

Another topic of active research is the nature of attentional enhancement in visual cortex, particularly in visual area V1. Many studies have found increased activity in anticipation of a stimulus in early visual cortex when attention is deployed. The anticipatory signals have been interpreted as changes in baseline firing rates, and there is some evidence that these baseline changes are the sole effect of attention in visual cortex (Murray, 2008). On the other hand, some studies have found evidence of delayed enhancement in V1 (Noesselt et al., 2002), suggesting that apart from effects preceding the stimulus, there is an additional effect of attention after the stimulus appears.

Bressler et al. (2008) present Granger causality results only during the anticipatory period, but it will be interesting to see whether the Granger causality changes once the stimulus has appeared. In their Figure 3C (http://www.jneurosci.org/cgi/ content/full/28/40/10056/F3), there are some general patterns to the Granger causality results; for example, areas V4 and VP show more top-down influences than areas $\mathrm{V} 1$ and $\mathrm{V} 2$. If baseline changes are the only effect of attention in visual cortex, the pattern of top-down Granger causality should not change after the stimulus appears.

Alternatively, attention may rely on feedforward stimulus information reaching some cortical level, which feeds back to early visual areas. For example, Noesselt et al. (2002) found that responses were initially unaffected by attention in V1, but were enhanced $\sim 140-250 \mathrm{~ms}$ after the onset of the stimulus-driven response. They first observed effects of attention in extrastriate cortex, and they proposed two explanations for a delayed effect in V1. In the first explanation, feedback signaling from extrastriate neurons to $\mathrm{V} 1$ neurons is responsible for the delayed V1 enhancement. In the second explanation, extrastriate cortex projects to some other brain region that affects the responses in V1. Granger causality analyses applied to the poststimulus period may be able to distinguish between these alternatives.

The results of Bressler et al. (2008) are consistent with the hypothesis that attentional control regions in the FEF and IPS are responsible for the changes in visual cortex activity in anticipation of a stimulus. While previous studies have suggested this role for the FEF and IPS, Bressler et al. (2008) provide the first evidence that activity in the FEF and IPS predicts subsequent activity in human visual cortex under physiological conditions. The data support a model in which the process of spatial selection is initiated in the FEF, but the precise circuitry of attentional control remains controversial. Finally, Granger causality measures between brain areas in fMRI time series may provide future insights into the control of visual attention, as well as other cognitive processes.

\section{References}

Bressler SL, Tang W, Sylvester CM, Shulman GL, Corbetta M (2008) Top-down control of human visual cortex by frontal and parietal cortex in anticipatory visual spatial attention. J Neurosci 28:10056-10061.

Green JJ, McDonald JJ (2008) Electrical neuroimaging reveals timing of attentional control activity in human brain. PLoS Biol 6:e81.

Grent-'t-Jong T, Woldorff MG (2007) Timing and sequence of brain activity in top-down control of visual-spatial attention. PLoS Biol 5:e12.

Murray SO (2008) The effects of spatial attention in early human visual cortex are stimulus independent. J Vis 8:2.1-11.

Noesselt T, Hillyard SA, Woldorff MG, Schoenfeld A, Hagner T, Jäncke L, Tempelmann C, Hinrichs H, Heinze HJ (2002) Delayed striate cortical activation during spatial attention. Neuron 35:575-587.

Roebroeck A, Formisano E, Goebel R (2005) Mapping directed influence over the brain using granger causality and fMRI. Neuroimage 25:230-242.

Sylvester CM, Shulman GL, Jack AI, Corbetta M (2007) Asymmetry of anticipatory activity in visual cortex predicts the locus of attention and perception. J Neurosci 27:14424-14433. 